\section{Dementia \\ and Geriatric}

Cognitive Disorders

Dement Geriatr Cogn Disord 2016;41:220-232

\title{
A Review of Pain Prevalence in Alzheimer's, Vascular, Frontotemporal and Lewy Body Dementias
}

\author{
Janine van Kooten ${ }^{a, b}$ Tarik T. Binnekade ${ }^{d}$ \\ Johannes C. van der Wouden ${ }^{a, b}$ Max L. Stek ${ }^{b, c}$ Erik J.A. Scherder ${ }^{d}$ \\ Bettina S. Husebø $\varnothing^{e}$ Martin Smalbrugge ${ }^{a, b}$ Cees M.P.M. Hertogh ${ }^{a, b}$ \\ a Department of General Practice and Elderly Care Medicine, ${ }^{b} E M G O+$ Institute for \\ Health and Care Research, and ${ }^{\mathrm{C} G G Z}$ InGeest/Department of Psychiatry, VU University \\ Medical Center, and ${ }^{d}$ Department of Clinical Neuropsychology, VU University,

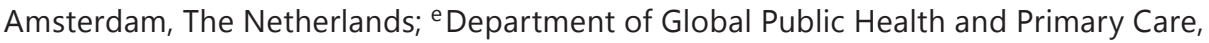 \\ Center for Elderly and Nursing Home Medicine, University of Bergen, Bergen, Norway
}

\section{Key Words}

Pain - Alzheimer's disease - Vascular dementia - Frontotemporal dementia - Lewy body dementia

\begin{abstract}
Background: Numerous studies have reported on pain in dementia. It has been hypothesized that pain perception differs between dementia subtypes, and therefore, the prevalence of pain differs between dementia subtypes. However, there remains a paucity of evidence on the differences in the prevalence of pain in different dementia subtypes. This review aimed to determine the prevalence of pain for the major dementia subtypes: Alzheimer's disease (AD), vascular dementia (VaD), frontotemporal dementia (FTD) and dementia with Lewy bodies (DLB). Summary: We found 10 studies that met our inclusion criteria. Most of these studies reported on AD; studies reporting the prevalence of pain in people with DLB were scarce, and for FTD, we found no studies. The sample-weighted prevalence of pain could only be calculated for $A D, V a D$ and mixed dementia: $A D$ 45.8\% (95\% confidence interval, CI: 33.4-58.5\%), VaD 56.2\% (95\% CI: 47.7-64.4\%) and mixed dementia 53.9\% (95\% CI: 37.4-70.1\%). Key Messages: Studies investigating the prevalence of pain in dementia subtypes were scarce; however, we found a high prevalence of pain in dementia without significant differences between the dementia subtypes. More studies are required to draw firm conclusions on the differences in the prevalence of pain between dementia subtypes.




\section{Introduction}

Pain in people with dementia is a common problem, and in view of the demographic changes and aging world population, more people can be expected to suffer from both dementia and a painful condition in the near future [1-4]. The prevalence of pain in people with dementia has been estimated to be approximately $50 \%$, and it has been suggested that the prevalence of pain differs between dementia subtypes [5].

Neuropathological changes differ between dementia subtypes, and these are held responsible for the decline in function as well as for alterations in pain perception [3, 5]. For example, pain perception in Alzheimer's disease (AD) may be altered because of a loss of grey matter, which leads to an increased pain tolerance without a change in pain threshold $[6,7]$. Pain experience in vascular dementia $(\mathrm{VaD})$ may increase because of white matter lesions, while pain experience in frontotemporal dementia (FTD) may decrease because atrophy predominates in the frontal cortex [8]. This area is related to emotional states and motivation, which may explain the frequently reported loss of awareness of pain in people with FTD [9]. Pain perception in dementia with Lewy bodies (DLB) may be altered due to brain damage caused by Lewy bodies and cortical atrophy [10]. As our understanding of the underlying neuropathology has increased, it seems worthwhile to take into account the dementia subtype in the management of pain in people with dementia. However, the prevalence of pain has not been determined systematically for each dementia subtype $[8,11]$.

Our objectives were to determine the prevalence of pain per dementia subtype in published studies that focused specifically on one of the four major dementia subtypes (i.e. $\mathrm{AD}, \mathrm{VaD}, \mathrm{FTD}$ and $\mathrm{DLB}$ ) in samples from both the community and long-term care facilities (LTCFs) [12]. Because of the differences in neuropathological changes, we hypothesized that the prevalence of pain differs between the four dementia subtypes.

\section{Methods}

Search Strategy

We searched MEDLINE, EMBASE, CINAHL and PsycINFO for articles about patients with a known dementia subtype and pain. Databases were searched from inception until August 2014. The search strategy consisted of a combination of free terms and medical subject headings (e.g. MeSH) relating to pain and the 4 commonest subtypes of dementia (i.e. AD, VaD, FTD and DLB). Articles were screened for dementia subtype and pain in the title or abstract text. Additionally, we identified studies by handsearching reference lists of published literature reviews on pain in dementia and studies that met our inclusion criteria. There were no language restrictions.

\section{Selection of Studies}

For inclusion, studies had to meet the following criteria: presenting primary data including prevalence of pain in patients with a dementia subtype diagnosis. Studies were independently screened for eligibility on titles and abstract by two reviewers (T.T.B./J.v.K.), and after both reviewers reached consensus, they reviewed the full text of the thus selected papers. Studies that reported pain for both cognitively intact and cognitively impaired patients were included if they reported the results separately to enable data extraction of pain prevalence per dementia subtype. We excluded studies that used pain as an inclusion or exclusion criterion, studies on induced pain (e.g. [13]), case reports or case series. Authors were contacted when studies reported one or more dementia subtypes, but did not display the results of the subtypes separately. Papers were subsequently excluded if the authors were not able to provide the required data or did not respond to our request. 
van Kooten et al.: A Review of Pain Prevalence in Alzheimer's, Vascular, Frontotemporal and Lewy Body Dementias

Data Extraction and Synthesis

Two reviewers independently extracted data on study design, setting, population, dementia subtype, prevalence of pain and measurement instruments. For one study, published in Spanish, data was extracted by only one reviewer, who had knowledge of the Spanish language.

We assessed the external and internal validity of the study findings using the Methodological Evaluation of Observational Research (MORE) checklist, which was adapted to the specific research question [14]. External validity is defined as the extent to which the results of a study can be generalized to the target population. For this study, the checklist comprised the following questions:

- Was the sampling frame a close representation of the target population?

- Was an appropriate case definition used for dementia (subtype)?

- Was dementia stage measured in the target population?

- Was some form of random selection used to select the sample?

- Was the likelihood of nonresponse bias minimal?

Internal validity is defined as the possible amount of error in measuring the conditions and was addressed by the following question:

- Was an acceptable pain assessment tool used for the assessment of pain?

For each criterion, three options were possible: '+' = low risk of bias, '-' = possible risk of bias or '?' = risk of bias unclear (due to poor reporting). Each study was rated independently by two reviewers. A total score was calculated as the sum of the individual criteria using a score of ' 1 ' for low risk of bias and a score of ' 0 ' for possible or unclear risk of bias. Scores for the criterion 'nonreponse' were only reported in two studies; therefore, we decided to exclude this criterion from the total score. We independently extracted information and scores for validity, and all discrepancies were resolved by consensus. We qualitatively evaluated individual studies for similarities and differences in study design and results.

Analysis

The sample-weighted averages with $95 \%$ confidence intervals (CI) were calculated for subgroups that consisted of $\geq 100$ participants. We used a random-effects model if the $\mathrm{I}^{2}$ statistic was greater than $50 \%$, and a fixed-effect model if the $\mathrm{I}^{2}$ statistic was under $50 \%[15,16]$. Statistical analyses were performed using MedCalc for Windows, version 5.5, 32 bit (MedCalc Software, Ostend, Belgium). In subgroups consisting of $<100$ participants, we confined ourselves to descriptive analyses.

\section{Results}

The initial electronic search yielded 2,374 hits: 475 from MEDLINE, 1,151 from EMBASE, 311 from CINAHL and 437 from PsycINFO. After removing duplicates, a total of 1,709 articles were screened for eligibility based on title and abstract. Another 4 publications were identified by reference checking and a total of 85 underwent full-text review (fig. 1). We contacted 16 authors for further information and five of them provided additional information. The main reasons for exclusion were no data on prevalence of pain and no data on dementia subtype. Eventually, 10 studies met all inclusion criteria. The characteristics and main findings of the included studies are described in table 1.

All studies were published in the last 10 years. Eight studies were conducted in Europe [17-25] and two studies in the USA [26, 27]. Five studies examined outcomes in nursing homes [23-27], whereas five studies examined outcomes in community-dwelling people or patients recruited from outpatient clinics [17, 18, 20-22]. Five studies were cross-sectional $[17,21,22,24,25,27]$, two longitudinal observational studies [19, 23], one was a case-control study [18], one a retrospective cohort study [26] and one a randomized controlled trial [20]. The mean subject age ranged from 70 to 86 years. Three studies focused solely on people with $\mathrm{AD}[17,21,22,27]$, five studies included people with $\mathrm{AD}, \mathrm{VaD}$, mixed pathologies and/or DLB $[20,23-26]$, one study was a case-control study of AD and DLB [18] and one study included dementia subtypes based on motor neuron symptoms (i.e. parkinsonian syndromes) [19]. No study reported the prevalence of pain specific to people with FTD. 


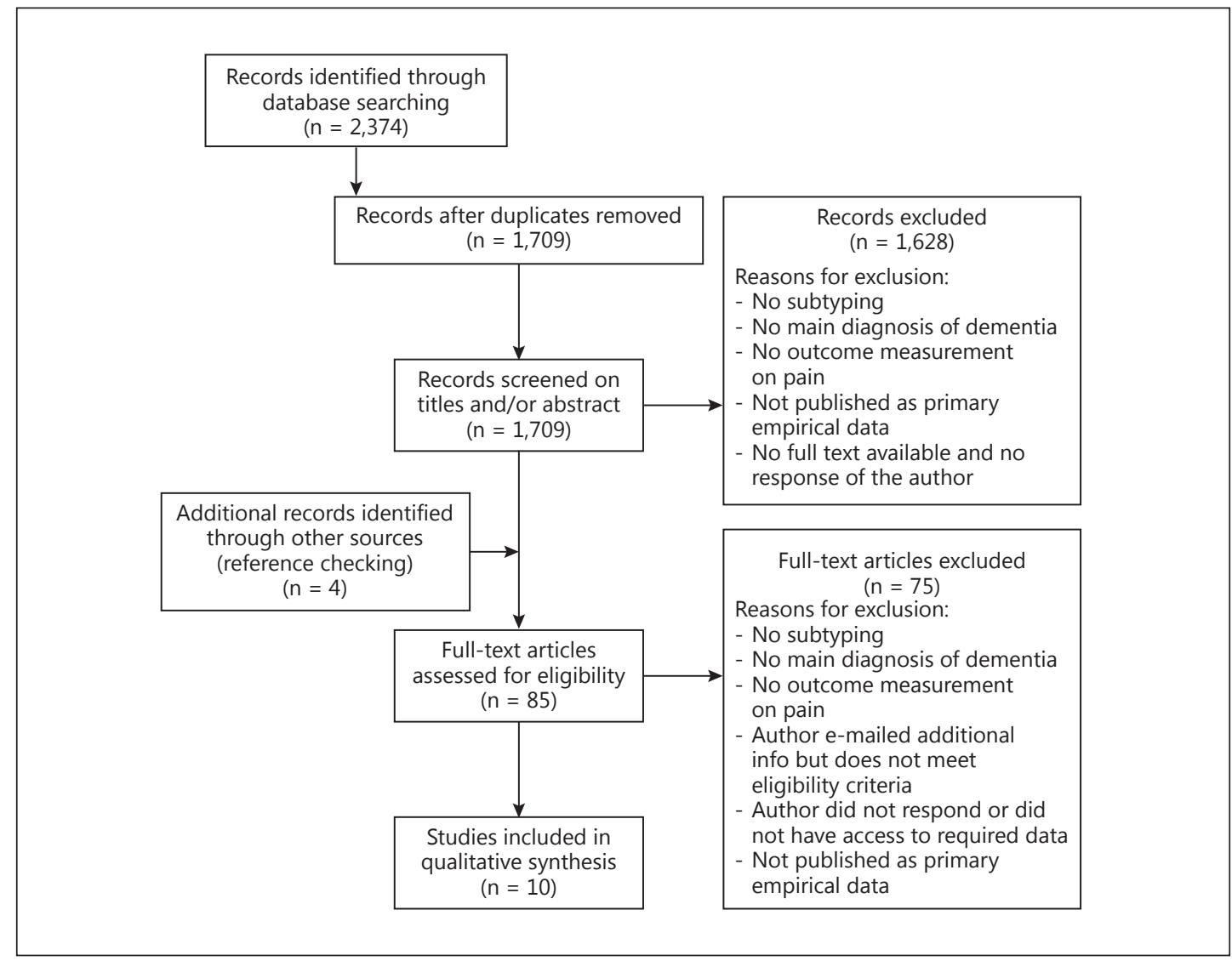

Fig. 1. Study selection process.

Different instruments for pain measurement were reported; four studies used the EQ-5D $[17,18,20,21]$, two studies the Minimum Data Set (MDS) [25, 27], one study the MobilizationObservation-Behaviour-Intensity-Dementia-2 (MOBID-2) Pain Scale [24], one study the Discomfort Behavioural Scale (DBS) [26], one study a questionnaire that included pain domains [19], and in one study, the physician assessed if pain was present [23].

\section{Risk Assessment}

Most studies did not meet all criteria for validity for observational studies. The nonreponse rate was only reported for two studies. With respect to the external validity, the risk of bias was considered possible in most studies, and the most frequent source of bias was found in the random selection of participants. Additionally, with respect to the internal validity, the risk of bias of the measurement of pain was considered low in most of the studies. Only two studies used a nonspecific measurement tool, and in one study, a proxy was asked to fill in or help to fill in the EQ-5D (table 2).

\section{Overall Sample}

The 10 published reports provided information on 51,810 participants. Except for one study, which provided information on 49,627 patients, the sample size ranged from 48 to 929. Most studies included more women than men, except for the studies by Colosimo et al. [19] and Boström et al. [18], which both included people with DLB. The mean age of the 
Dementia

Cognitive Disorders
Dement Geriatr Cogn Disord 2016;41:220-232 www.karger.com/dem

van Kooten et al.: A Review of Pain Prevalence in Alzheimer's, Vascular, Frontotemporal and Lewy Body Dementias

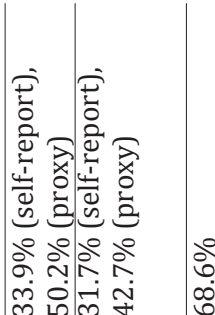

䒕

ㄴ.

蝠
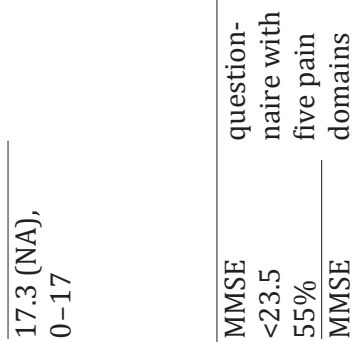

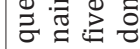

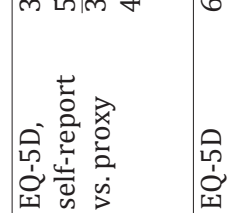

\section{$\sum_{\Sigma}^{n} \tilde{5}$

底

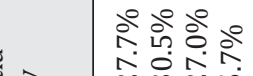
ปิ

के นก

$\frac{\pi}{z}$



$\stackrel{0}{\circ}$

$\Xi \dot{2}$ 胥 完



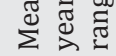

$\stackrel{\circ}{\circ \infty}$

일





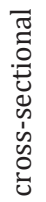
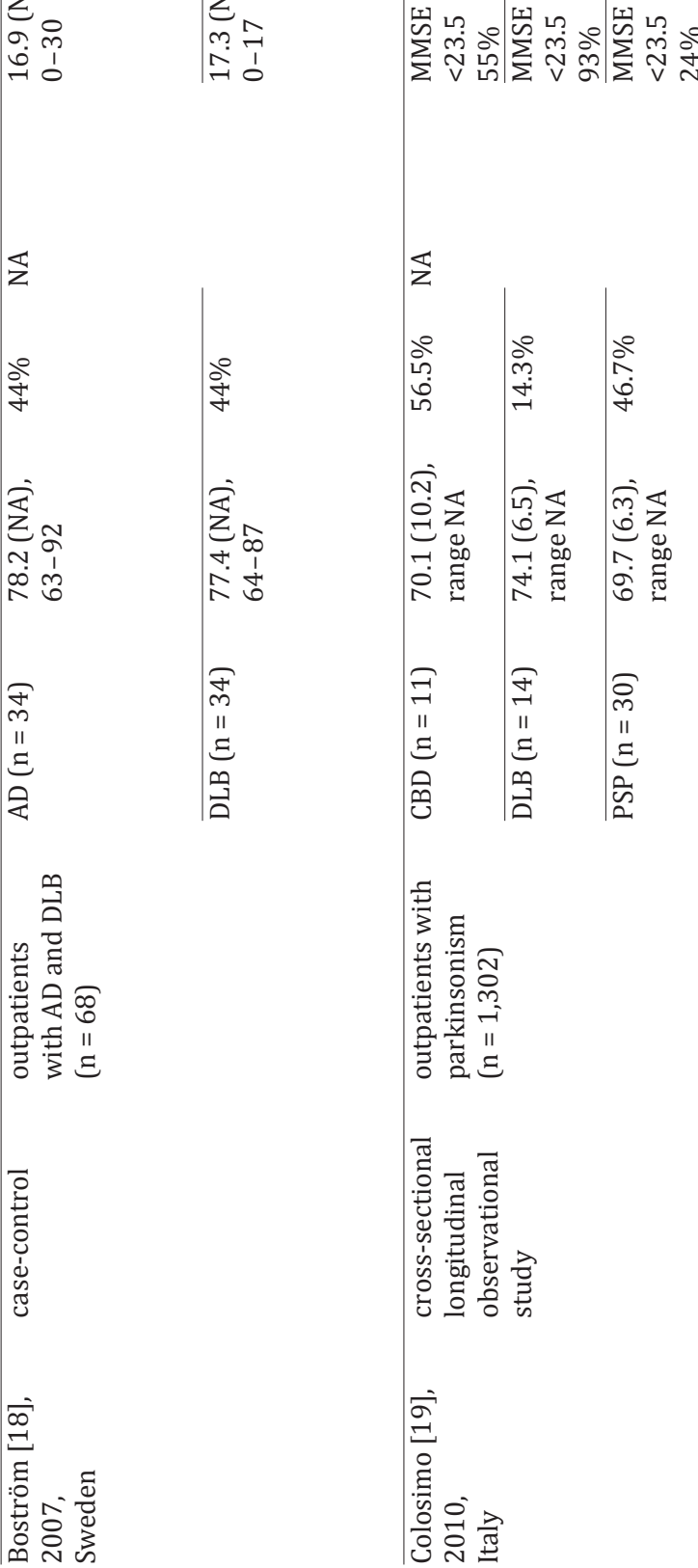

i্c




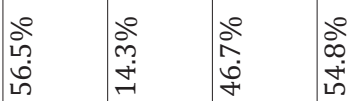

:

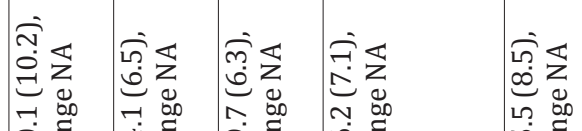



象

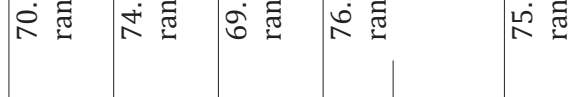

ᄏ
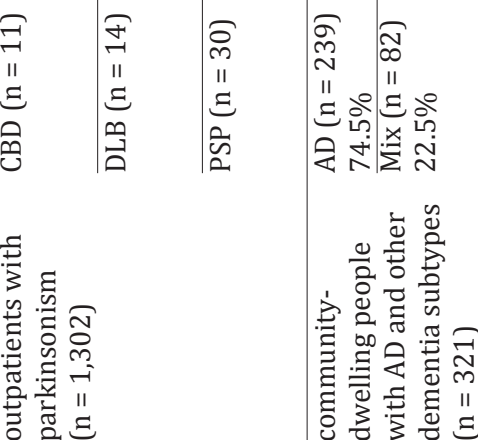

命

웅.



들



\section{욤}

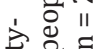

20

寻导

总究

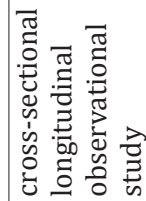

बे

이

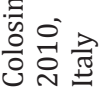




van Kooten et al.: A Review of Pain Prevalence in Alzheimer's, Vascular, Frontotemporal and Lewy Body Dementias

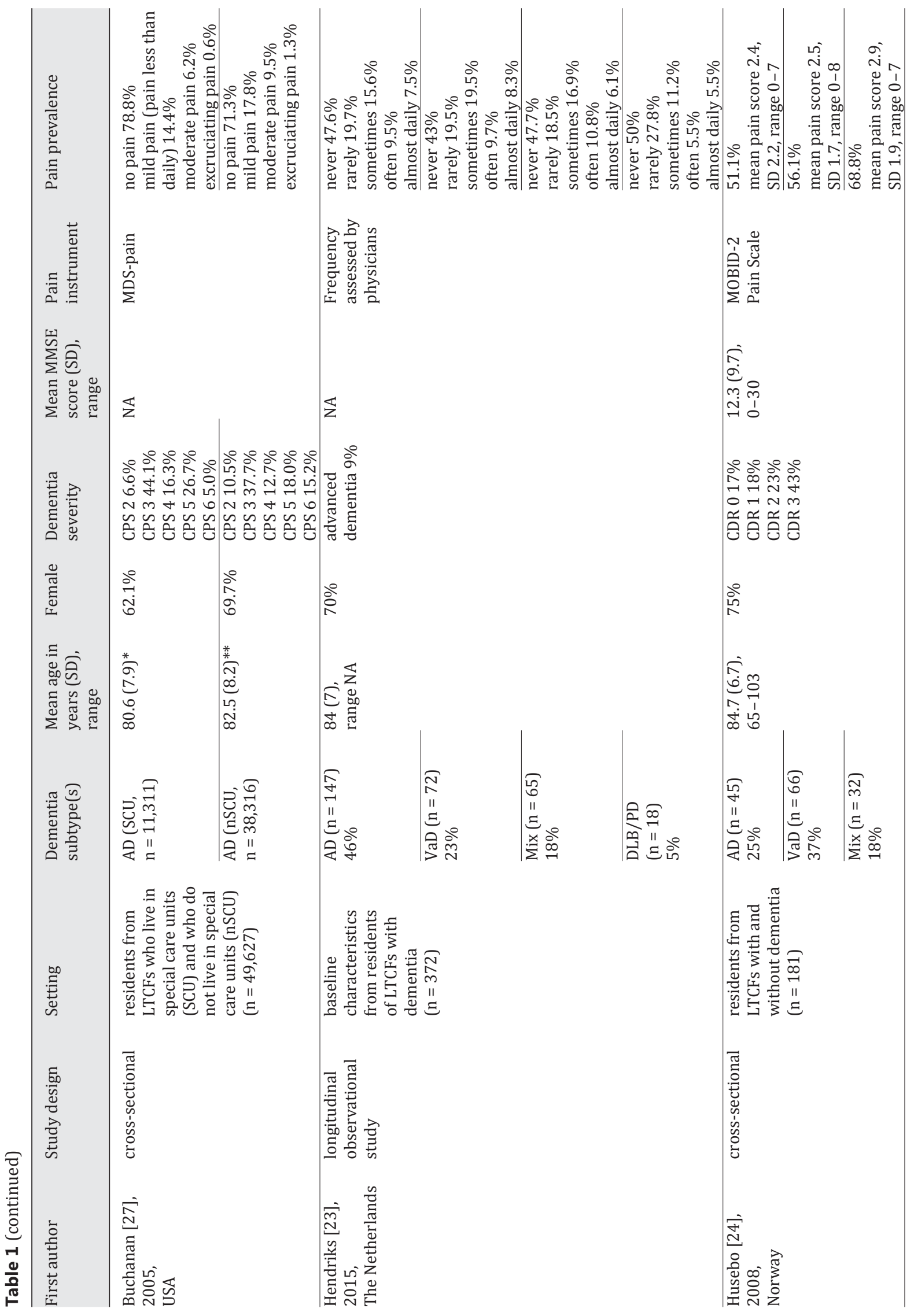


Dementia

and Geriatric
Cognitive Disorders
Dement Geriatr Cogn Disord 2016;41:220-232

O www.karger.com/dem

van Kooten et al.: A Review of Pain Prevalence in Alzheimer's, Vascular, Frontotemporal and Lewy Body Dementias

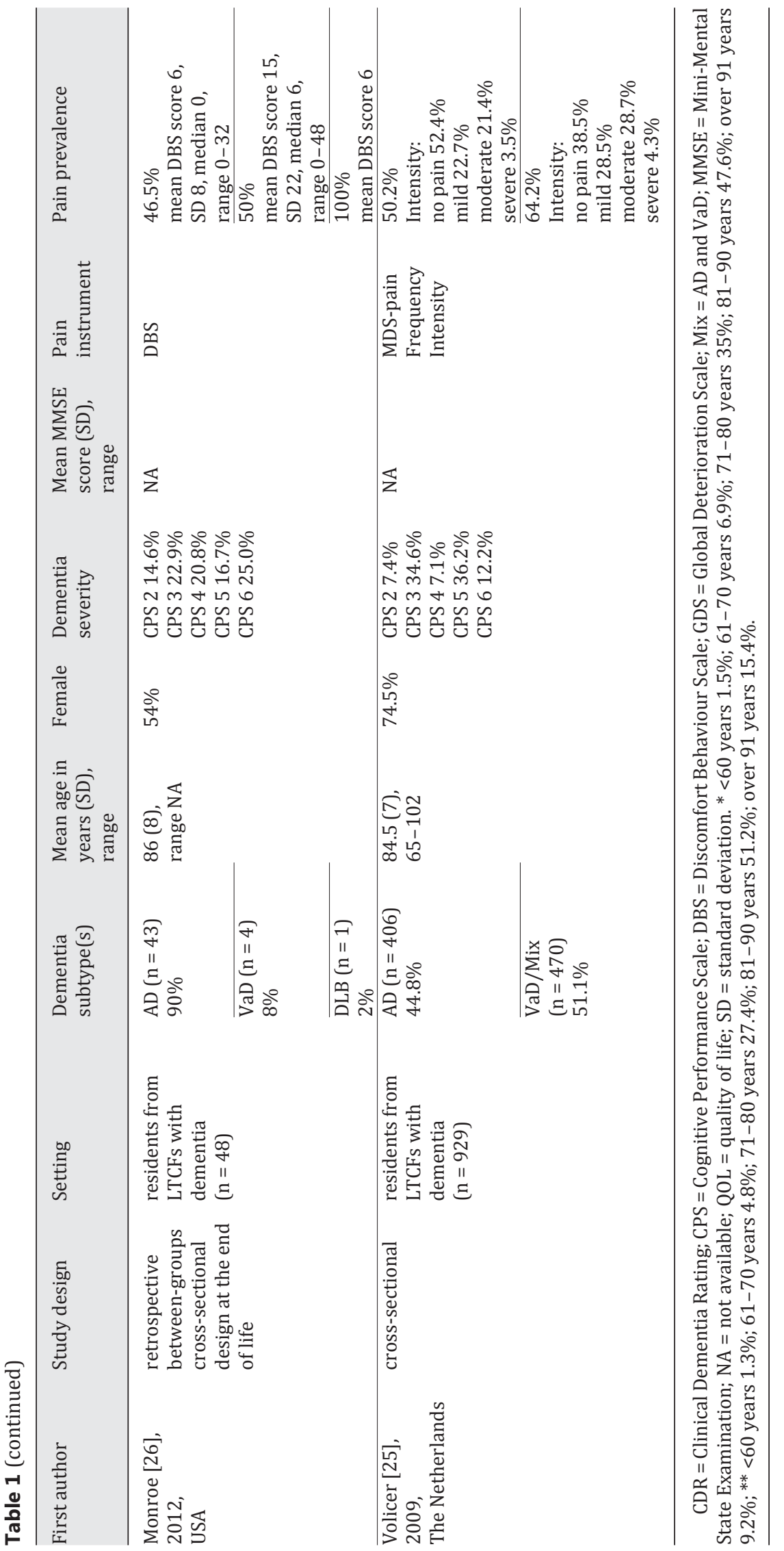


Dementia

Cognitive Disorders
Dement Geriatr Cogn Disord 2016;41:220-232 www.karger.com/dem

van Kooten et al.: A Review of Pain Prevalence in Alzheimer's, Vascular, Frontotemporal and Lewy Body Dementias

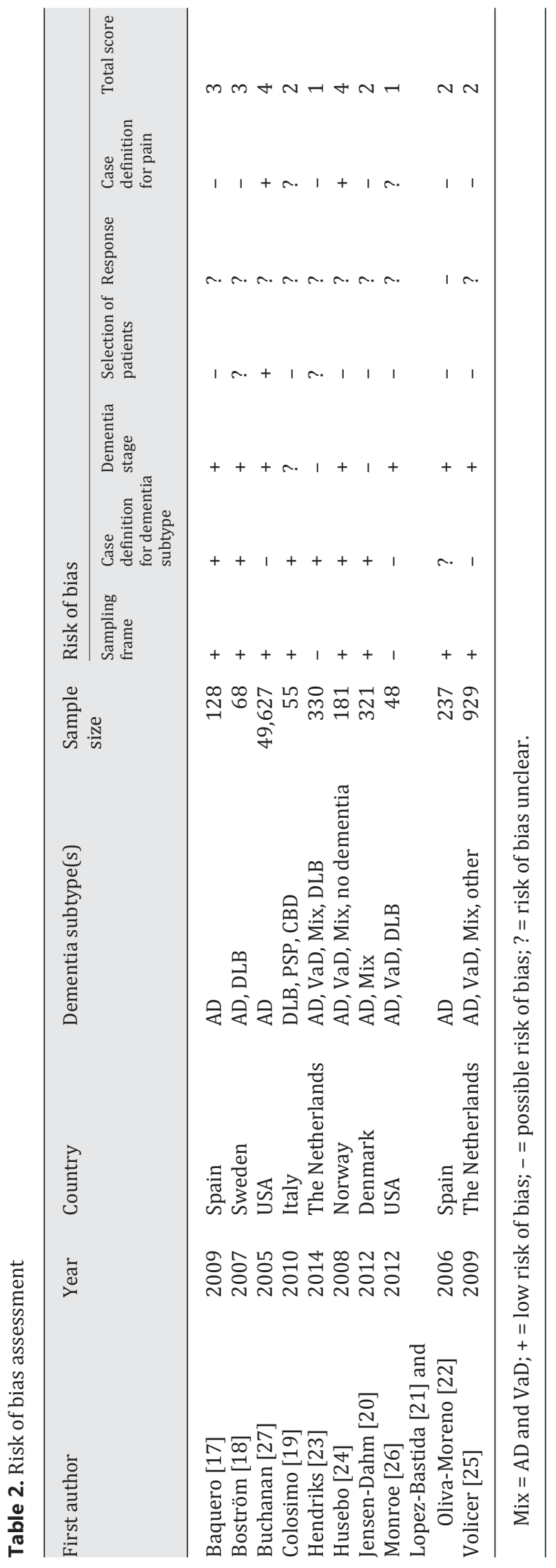


van Kooten et al:: A Review of Pain Prevalence in Alzheimer's, Vascular, Frontotemporal and Lewy Body Dementias

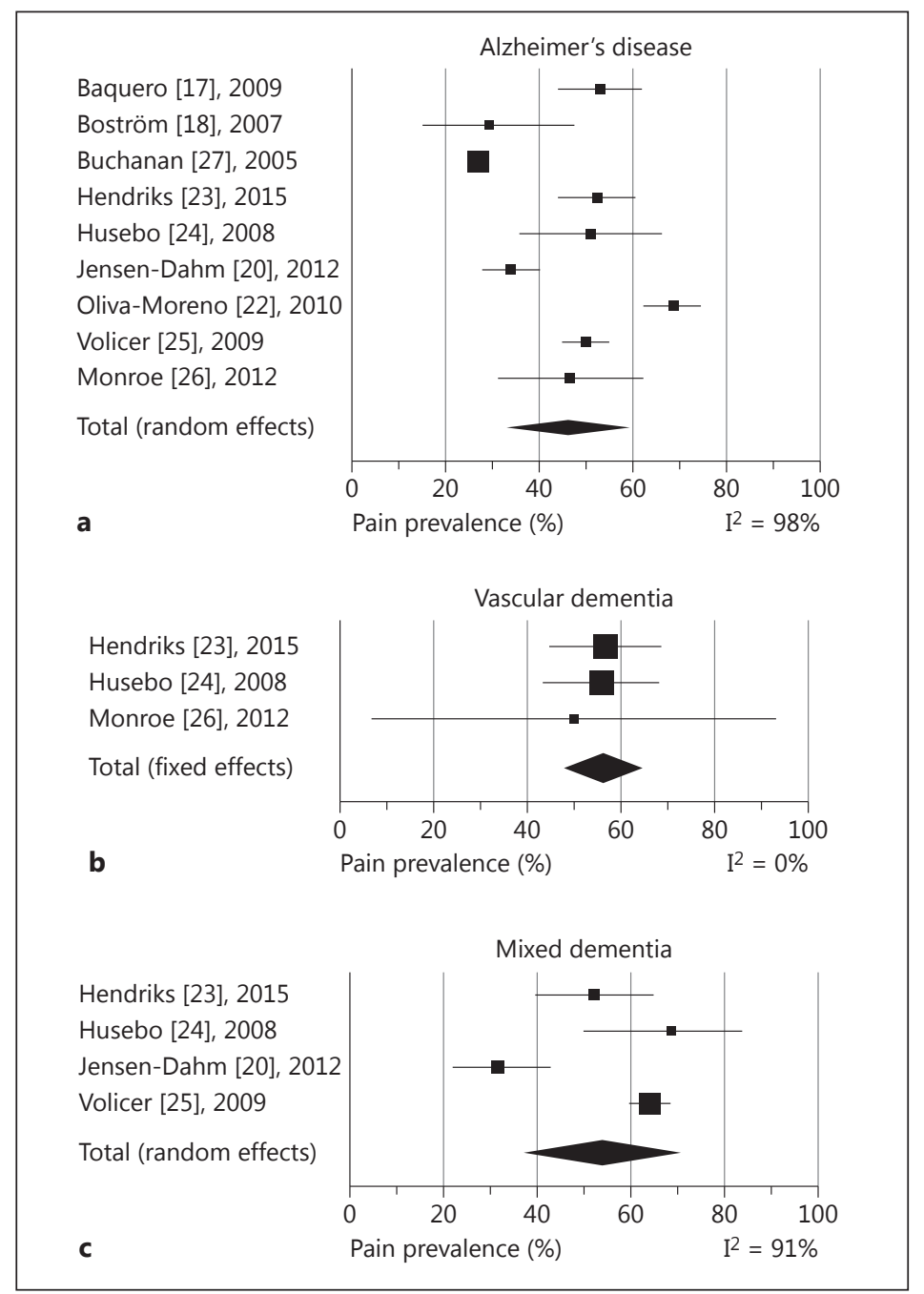

participants in LTCFs was above 80 years, and the mean age of the other participants (i.e. outpatient and community-dwelling people) was $<80$ years. Nine studies provided crosssectional data on pain prevalence, one study measured pain at the end of life [26].

\section{Prevalence of Pain in Dementia Subtypes}

Nine studies reported the proportion of pain in people with AD [17, 18, 27]. Eight studies used cross-sectional data and one study reported data from a case-control study. The sample size of Alzheimer patients was 50,911, including one study with 49,627 patients, and age ranged from 48 to 103 years. The mean prevalence of pain in AD in our study was $45.8 \%$ (95\% CI: 33.4-58.5\%) with a substantial heterogeneity ( $\mathrm{I}^{2}=98 \%$; fig. $\left.2 \mathrm{a}\right)$.

Three studies reported on pain prevalence solely in people with $\operatorname{VaD}[22,23]$. These studies collected data on more than one dementia subtype and were based on cross-sectional data from studies carried out in LTCFs. The sample of people with VaD was a homogeneous subgroup $\left(\mathrm{I}^{2}=0 \%\right)$ that consisted of 142 participants, and the mean prevalence of pain in $\mathrm{VaD}$ was $56.2 \%$ (95\% CI: 47.7-64.4\%; fig. $2 \mathrm{~b}$ ).

We found mixed dementia ( $\mathrm{AD}$ and $\mathrm{VaD}$ ) to be reported frequently as a separate entity next to $\mathrm{AD}$ and $\mathrm{VaD}$, and therefore, we presented pain prevalence for mixed dementia sepa- 
van Kooten et al.: A Review of Pain Prevalence in Alzheimer's, Vascular, Frontotemporal and Lewy Body Dementias

rately. Four studies reported on mixed dementia; three studies used cross-sectional data and one study provided data on prevalence using the baseline data of a randomized controlled trial [20,23-25]. The sample of patients with mixed dementia was 649, and the mean prevalence of pain in mixed dementia was 53.9\% (95\% CI: 37.4-70.1\%) with a substantial heterogeneity $\left(I^{2}=91 \%\right.$; fig. $\left.2 c\right)$.

Three studies reported on DLB, whereas one study combined Parkinson disease (PD) and DLB into one group. After contact with the author, it appeared that they could not be untangled. In their sample were 18 people, and nine (50\%) of them suffered from pain [23]. The study by Boström et al. [18] was a case-control study that included 34 outpatients with AD and 34 outpatients with DLB. The prevalence of pain $(n=24)$ in DLB was $70 \%$ and in those with AD ( $n=32$ ) 30\% [18]. The study by Colosimo et al. [19] collected data on nonmotor symptoms in parkinsonian syndromes, and they included 14 people with DLB, of whom seven (50\%) indicated that they were in pain. We did not find enough studies to calculate a sampleweighted prevalence for DLB.

We found no studies on FTD, but we retrieved one study concerning two conditions closely related to FTD: corticobasal degeneration (CBD) and progressive supranuclear palsy (PSP) $[19,28]$. Colosimo et al. [19] collected data from 11 patients with CBD and from 30 patients with PSP. Among those with CBD, 4 patients (37\%) indicated that they had pain, and among those with PSP, 7 patients expressed pain (23\%) [19].

\section{Discussion}

In this systematic review, we reviewed the current literature on pain in dementia to present the prevalence of pain per dementia subtype, although we found not enough studies to report a sample-weighted prevalence for each dementia subtype. We identified 10 studies that reported on pain prevalence in $\mathrm{AD}, \mathrm{VaD}$, DLB or mixed dementia (AD and VaD). We found no studies that reported on pain prevalence in FTD.

We calculated the sample-weighted prevalence, which was $45.8 \%$ (95\% CI: 33.4-58.5\%) for $\mathrm{AD}, 56.4 \%$ (95\% CI: 47.8-64.8\%) for $\mathrm{VaD}$ and 53.9\% (95\% CI: 37.4-70.1\%) for mixed dementia. Our findings could not confirm the hypothesis that pain prevalence differs significantly between the different dementia subtypes.

The sample-weighted prevalence for people with $\mathrm{AD}, \mathrm{VaD}$ and mixed dementia are comparable to the estimated pain prevalence of 50\% that Zwakhalen et al. [29] found in their review on pain in dementia (2005); however, in their review, pain was not reported per dementia subtype. As AD, VaD and mixed dementia together account for two thirds of all dementia cases, our findings seem to be in line with those of previous studies [29, 30].

The wide range of pain prevalence might be explained by the heterogeneity in study designs, settings, patient characteristics and definition of pain. For example, in one study, pain was reported twice: one self-report and one proxy rating. As self-report is the golden standard in pain assessment [31], we decided to extract the self-reported data, but prevalence measured by self-report was lower than measured by proxy. This is in line with the findings of Scherder and van Manen [32], who found that self-reported pain in patients with AD was lower than pain observed by a nursing assistant. This may indicate that people with $\mathrm{AD}$ do not suffer from pain to the extent that their caregivers expect them to do or that the prevalence of pain may depend on the methods and data sources used as well as the time frame of pain detection $[31,33]$.

This review is not without limitations. To begin with, literature on this specific topic seems to be scarce; only 10 studies on pain in dementia subtypes fulfilled our inclusion criteria, and these studies showed substantial heterogeneity in study design. For example, prevalence of pain was usually not the main objective of the studies included in our review. In addition, we 
found no studies on pain in FTD. This lack of studies could be explained by several epidemiological factors; for instance, FTD is relatively more prevalent in a younger population, whereas pain is more prevalent in the older population [4]. A specific reason for the lack of studies in DLB could be that these patients may be studied within trials assessing pain in PD, as Lewy body neuropathology is a key feature of PD and prevalence of pain in PD is estimated to be between 40 and $60 \%[34,35]$. Furthermore, we found that the methods of pain assessment varied widely, and many of the used instruments were not specifically designed for the population, for example the EQ-5D, which is an instrument to measure quality of life and only incorporates one pain item. We found the use of nonspecific assessment tools to be more common than the use of dementia-specific pain assessment tools. On the one hand, this was surprising, as more than 30 specific measurement instruments have been designed to measure pain especially in people with dementia $[29,36]$, but on the other hand, this was not surprising, as the main objectives of the included studies did not include prevalence of pain. Another limitation that should be noted is that we could not correct for the influence of comorbid pain conditions, as most of the included studies did not report comorbidity.

Our findings correspond to the previously reported prevalence of pain in people with dementia of 50\%; also, our analyses showed that pain prevalence in dementia subtypes was not statistically significantly different. However, it cannot be completely ruled out that the prevalence of pain differs between the four dementia subtypes as a consequence of the differences in neuropathological changes, as we did not identify studies on pain in FTD and DLB, and we were not able to control for confounding by dementia severity and differences in pain caused by comorbidity in subtypes of dementia.

\section{Conclusion}

This is the first study that used a systematic approach to review the available literature on pain prevalence in dementia subtypes and calculated the sample-weighted prevalence of pain in patients with $\mathrm{AD}, \mathrm{VaD}$, and mixed dementia. However, we found not enough studies to calculate a sample-weighted prevalence for DLB, and we found no studies that reported on pain prevalence in FTD. The pain prevalence in FTD and DLB has not been investigated extensively, which may be due to epidemiological issues, such as the higher prevalence of $\mathrm{AD}, \mathrm{VaD}$, and mixed dementias as well as methodological issues. Whilst this study could not show a difference in pain prevalence for the different dementia subtypes, this review underlines that pain is reported frequently in people with dementia, and we recommend uniformity in pain assessment. More well-designed studies are required to draw firm conclusions on differences in prevalence of pain between dementia subtypes as a consequence of the differences in neuropathological changes. These studies should take into account comorbidity as potential confounder and focus especially on people with DLB and FTD, as both groups are underrepresented in earlier research. Research should also focus on the causes, course and characteristics of pain. Eventually, pain prevalence and its possible impact on quality of life should be a topic of future research.

\section{Acknowledgements}

We would like to thank Bettina Husebø, Todd Monroe, Elizabeth Londos, Ladislav Volicer, and Simone Hendriks for providing us with additional data for the present review. This study was funded by Amstelring, Alzheimer Nederland, Fonds Nuts Ohra, Roomsch Catholijk Oude Armen Kantoor (RCOAK), Stichting Beroepsopleiding Huisartsen (SBOH) and Stichting Henriëtte Hofje. 
van Kooten et al.: A Review of Pain Prevalence in Alzheimer's, Vascular, Frontotemporal and Lewy Body Dementias

\section{Disclosure Statement}

The authors declare that they have no competing interests.

\section{References}

$\rightarrow 1$ Woolf AD, Vos T, March L: How to measure the impact of musculoskeletal conditions. Best Pract Res Clin Rheumatol 2010;24:723-732.

-2 Cook AK, Niven CA, Downs MG: Assessing the pain of people with cognitive impairment. Int J Geriatr Psychiatry 1999;14:421-425.

-3 Achterberg WP, Pieper MJ, van Dalen-Kok AH, de Waal MW, Husebo BS, Lautenbacher S, et al: Pain management in patients with dementia. Clin Interv Aging 2013;8:1471-1482.

4 Duncan R, Francis RM, Collerton J, Davies K, Jagger C, Kingston A, et al: Prevalence of arthritis and joint pain in the oldest old: findings from the Newcastle 85+ study. Age Ageing 2011;40:752-755.

5 Scherder EJ, Sergeant JA, Swaab DF: Pain processing in dementia and its relation to neuropathology. Lancet Neurol 2003;2:677-686.

6 Jensen-Dahm C, Werner MU, Dahl JB, Jensen TS, Ballegaard M, Hejl AM, et al: Quantitative sensory testing and pain tolerance in patients with mild to moderate Alzheimer disease compared to healthy control subjects. Pain 2014;155:1439-1445.

-7 Scherder E, Oosterman J, Swaab D, Herr K, Ooms M, Ribbe M, et al: Recent developments in pain in dementia. BMJ 2005;330:461-464.

-8 Scherder EJ, Slaets J, Deijen JB, Gorter Y, Ooms ME, Ribbe M, et al: Pain assessment in patients with possible vascular dementia. Psychiatry 2003;66:133-145.

-9 Bathgate D, Snowden JS, Varma A, Blackshaw A, Neary D: Behaviour in frontotemporal dementia, Alzheimer's disease and vascular dementia. Acta Neurol Scand 2001;103:367-378.

10 Burton EJ, McKeith IG, Burn DJ, Firbank MJ, O’Brien JT: Progression of white matter hyperintensities in Alzheimer disease, dementia with Lewy bodies, and Parkinson disease dementia: a comparison with normal aging. Am J Geriatr Psychiatry 2006;14:842-849.

11 Corbett A, Husebo B, Malcangio M, Staniland A, Cohen-Mansfield J, Aarsland D, et al: Assessment and treatment of pain in people with dementia. Nat Rev Neurol 2012;8:264-274.

12 van der Flier WM, Scheltens P: Epidemiology and risk factors of dementia. J Neurol Neurosurg Psychiatry 2005;76(suppl 5):v2-v7.

$\checkmark 13$ Alcolea D, Martinez-Lage P, Izagirre A, Clerigue M, Carmona-Iragui M, Alvarez RM, et al: Feasibility of lumbar puncture in the study of cerebrospinal fluid biomarkers for Alzheimer's disease: a multicenter study in Spain. J Alzheimers Dis 2014;39:719-726.

14 Shamliyan TA, Kane RL, Ansari MT, Raman G, Berkman ND, Grant M, et al: Development quality criteria to evaluate nontherapeutic studies of incidence, prevalence, or risk factors of chronic diseases: pilot study of new checklists. J Clin Epidemiol 2011;64:637-657.

15 Higgins JP, Thompson SG: Quantifying heterogeneity in a meta-analysis. Stat Med 2002;21:1539-1558.

16 Higgins JP, Thompson SG, Deeks JJ, Altman DG: Measuring inconsistency in meta-analyses. BMJ 2003;327: 557-560.

17 Baquero TM, Peset V, Burguera JA, Salazar-Cifre A, Bosca-Blasco ME, Del Olmo-Rodriguez A, et al: Quality of life in Alzheimer's disease. Rev Neurol 2009;49:337-342.

-18 Boström F, Jönsson L, Minthon L, Londos E: Patients with dementia with Lewy bodies have more impaired quality of life than patients with Alzheimer disease. Alzheimer Dis Assoc Disord 2007;21:150-154.

19 Colosimo C, Morgante L, Antonini A, Barone P, Avarello TP, Bottacchi E, et al: Non-motor symptoms in atypical and secondary parkinsonism: the PRIAMO study. J Neurol 2010;257:5-14.

-20 Jensen-Dahm C, Vogel A, Waldorff FB, Waldemar G: Discrepancy between self- and proxy-rated pain in Alzheimer's disease: results from the Danish Alzheimer Intervention Study. J Am Geriatr Soc 2012;60:12741278.

21 Lopez-Bastida J, Serrano-Aguilar P, Perestelo-Perez L, Oliva-Moreno J: Social-economic costs and quality of life of Alzheimer disease in the Canary Islands, Spain. Neurology 2006;67:2186-2191.

22 Oliva-Moreno J, Lopez-Bastida J, Worbes-Cerezo M, Serrano-Aguilar P: Health related quality of life of Canary Island citizens. BMC Public Health 2010;10:675.

23 Hendriks SA, Smalbrugge M, Galindo-Garre F, Hertogh CM, Van Der Steen JT: From admission to death: prevalence and course of pain, agitation, and shortness of breath, and treatment of these symptoms in nursing home residents with dementia. J Am Med Dir Assoc 2015;16:475-481.

24 Husebo BS, Strand LI, Moe-Nilssen R, Husebo S, Aarsland D, Ljunggren AE: Who suffers most? Dementia and pain in nursing home patients: a cross-sectional study. J Am Med Dir Assoc 2008;9:427-433.

25 Volicer L, Van Der Steen JT, Frijters DHM: Modifiable factors related to abusive behaviors in nursing home residents with dementia. J Am Med Dir Assoc 2009;10:617-622.

-26 Monroe T, Carter M, Feldt K, Tolley B, Cowan RL: Assessing advanced cancer pain in older adults with dementia at the end-of-life. J Adv Nurs 2012;68:2070-2078. 
27 Buchanan RJ, Choi M, Wang S, Ju H, Graber D: Nursing home residents with Alzheimer's disease in special care units compared to other residents with Alzheimer's disease. Dementia 2005;4:249-267.

28 Sieben A, Van Langenhove T, Engelborghs S, Martin JJ, Boon P, Cras P, et al: The genetics and neuropathology of frontotemporal lobar degeneration. Acta Neuropathol 2012;124:353-372.

29 Zwakhalen SM, Hamers JP, Berger MP: The psychometric quality and clinical usefulness of three pain assessment tools for elderly people with dementia. Pain 2006;126:210-220.

30 Kalaria RN, Maestre GE, Arizaga R, Friedland RP, Galasko D, Hall K, et al: Alzheimer's disease and vascular dementia in developing countries: prevalence, management, and risk factors. Lancet Neurol 2008;7:812-826.

31 Pautex S, Michon A, Guedira M, Emond H, Le LP, Samaras D, et al: Pain in severe dementia: self-assessment or observational scales? J Am Geriatr Soc 2006;54:1040-1045.

-32 Scherder E, van Manen F: Pain in Alzheimer's disease: nursing assistants' and patients' evaluations. J Adv Nurs 2005;52:151-158.

-33 Scherder E, Bouma AF, Slaets JF, Ooms MF, Ribbe MF, Blok AF, et al: Repeated pain assessment in Alzheimer's disease. Dement Geriatr Cogn Disord 2001;12:400-407

-34 Wasner G, Deuschl G: Pains in Parkinson disease - many syndromes under one umbrella. Nat Rev Neurol 2012; 8:284-294.

35 Borsook D: Neurological diseases and pain. Brain 2012;135:320-344.

-36 Zwakhalen SM, Hamers JP, Abu-Saad HH, Berger MP: Pain in elderly people with severe dementia: a systematic review of behavioural pain assessment tools. BMC Geriatr 2006; 6:3. 